\title{
DOE+: Um Aplicativo Móvel de Cunho Social para Agendamento de Doação de Sangue no Hemocentro Público de Alagoas
}

\author{
Marcílio Ferreira de Souza Júnior ${ }^{1}$ \\ ${ }^{1}$ CODAI - Universidade Federal Rural de Pernambuco (UFRPE) \\ São Lourenço da Mata - PE - Brasil \\ marcilio.souzajrafuepe.br
}

\begin{abstract}
The adoption of innovative and digital social technologies with the potential to mitigate the steady trend in blood donations has been one of the strategies developed in Brazil to face the challenges of increasing donor uptake and regular donations by citizens. DOE + app was developed at the Blood Center of Alagoas State, Brazil, which sought to increase donor loyalty, especially from young people, in an attempt to awaken society's awareness of the need for more citizenship and collaboration in maintaining a satisfactory blood supply to meet the population needs.
\end{abstract}

Resumo. A adoção de tecnologias sociais inovadoras e digitais com potencial para atenuar a tendência estacionária das doações de sangue no Brasil tem sido uma das estratégias desenvolvidas no enfrentamento dos desafios de aumentar a captação de doadores e a regularidade da doação pelos cidadãos. Com esta problemática em mente, foi desenvolvido e implantado no Hemocentro de Alagoas, um aplicativo para dispositivos móveis, denominado $D O E+$, que buscou potencializar a fidelização de doadores, principalmente do público jovem, na tentativa de despertar a consciência da sociedade para a necessidade de mais cidadania e colaboração na manutenção de um estoque de sangue satisfatório para o atendimento das necessidades da população.

\section{Introdução}

Os hemocentros brasileiros lidam com a captação, triagem e doação de sangue, norteados pela Política Nacional de Sangue e Hemoderivados do Ministério da Saúde, que busca garantir a autossuficiência do Brasil em hemocomponentes a partir de ações para ampliar a doação de sangue da população, conforme Decreto $n^{\circ}$ 3.990/2001 (denominado "Lei do Sangue"), bem como adotando tecnologias digitais nos serviços de saúde pública [BRASIL 2001; ESTRATEGIA SAÚDE DIGITAL 2017].

Estudos sobre a percepção dos doadores de sangue relacionados ao atendimento no processo de doação buscaram aproximar as unidades hemoterápicas aos seus contextos culturais [Sojka 2008; Araújo; Feliciano e Mendes 2011], fornecendo recomendações para o enfrentamento dos desafios de aumentar a captação de doadores e a regularidade da doação de sangue, sendo uma das alternativas emergentes a adoção de tecnologias digitais neste processo. Em uma situação de demanda crescente por sangue e hemoderivados, a tendência estacionária das doações no Brasil, cujo percentual 
identificado pelo Ministério da Saúde correspondeu à aproximadamente 1,70\% da população, atenua a insuficiência dos estoques dos hemocentros [BRASIL 2001]. Neste cenário, não se percebe diferença na situação de escassez dos bancos de sangue no Hemocentro de Alagoas (HEMOAL) em comparação aos demais hemocentros públicos do país [Hora 2008], que também têm enfrentado dificuldades para manter um estoque de sangue satisfatório e mínimo necessário para atender à demanda de sangue da rede pública.

Por outro lado, o benefício do uso da tecnologia de informação aplicada à saúde (e-health) difundiu-se bastante com a adoção das tecnologias móveis [Saccol e Reinhard 2007; Oliveira e Rodrigues Costa 2012], destacando-se os aplicativos (apps) que ganharam relevância com o avanço da área de mobile health [Ouhbi et al. 2015]. Com a disseminação dos apps, Moura (2009) descortinou o advento da "geração polegar", que é formada por jovens atraídos pelas tecnologias móveis, usando-as diariamente para comunicação e interação em redes sociais, sendo que diversos aplicativos móveis vêm surgindo com o intuito de atrair este público e explorar seu potencial no contexto da atenção hematológica.

Conforme dito anteriormente, em Alagoas, a deficiência na captação e fidelização de doadores de sangue também é um problema crônico, tal como nos centros de coleta de sangue em todo país, sendo que ao mesmo tempo é imprescindível conscientizar os doadores para a manutenção dos níveis de estoque de bolsas sanguíneas nos hemocentros. Para enfrentar esta problemática, no período de 2015 a 2018, foi desenvolvido pelo Instituto Federal de Alagoas (IFAL) uma inovação tecnológica em uma parceria singular entre a Secretaria de Estado da Saúde de Alagoas (SESAU), o HEMOAL e a Secretaria de Estado da Ciência, Tecnologia e Inovação (SECTI), através de um termo de cooperação técnica entre as quatro instituições, que culminou na implantação inédita, em hemocentro público, de um app de cunho social [Baumgarten 2006] para agendamento de doações de sangue, denominado DOE+.

O presente artigo visa discutir os resultados da implantação do $\mathrm{DOE}+$, que potencializou a captação e a fidelização de doadores, principalmente do público jovem, na tentativa de despertar a consciência da sociedade para a necessidade de mais cidadania e colaboração na manutenção de um estoque de sangue com nível estratégico no atendimento às necessidades da população do estado.

A seguir são apresentadas as seções que descrevem os trabalhos correlatos, os procedimentos metodológicos da pesquisa, os resultados concretos e as discussões pósimplantação do aplicativo móvel no hemocentro de Alagoas, finalizando com as conclusões e contribuições do estudo.

\section{Trabalhos Correlatos}

Em um levantamento internacional, Ouhbi et al. (2015) identificaram 188 apps de uso livre com foco na doação de sangue, selecionando, dentre eles, 169 para análise em profundidade. Os resultados apresentados mostraram que a maioria dos aplicativos selecionados foi desenvolvido para o sistema operacional Android e objetivavam ajudar os usuários a procurar potenciais doadores de sangue. 
No Brasil, há registros de várias iniciativas para incentivar a doação de sangue via dispositivos móveis [Silva 2016], mas muitas já foram descontinuadas por motivos desconhecidos, como o projeto "Hemoliga" e a solução "Hemogram", que obtiveram projeção na mídia jornalística, mas cujos sites estão desativados atualmente. Outros aplicativos identificados como relevantes em uma busca no Google Scholar usando as palavras-chaves "aplicativo" e "doação de sangue" foram: Blooder (disponível para algumas regiões dos estados de São Paulo e Rio de Janeiro, mas não está atrelado a nenhum hemocentro); Eu Curto Doar (os doadores precisam efetuar um cadastro básico informando o tipo de sangue e a data limite para receber doações); Doe Sangue Mobile (conta com um sistema de localização de usuários que se encontram a um raio de até $100 \mathrm{~km})$.

No âmbito acadêmico, Orlando Silva Júnior e Pereira (2017) desenvolveram uma plataforma eletrônica para auxiliar na captação de doadores de sangue, com intuito de contribuir para o gerenciamento, incentivo e conscientização sobre o tema, além de centralizar pedidos de doação de sangue em todo o Brasil. Entre as principais funcionalidades disponibilizadas pelo aplicativo \#Partiudoarsangue estão: cadastro de doadores, mapa contendo a localidade dos hemocentros, campanhas, pedidos de doação de sangue e notificação de doadores. Contudo, diferentemente do presente estudo, o foco não eram os hemocentros públicos como gestor final da aplicação.

Silva e Barbosa de Araújo (2017) projetaram um software, denominado lupdonate, que serviu como um canal de apoio aos doadores. A arquitetura do sistema possui um módulo para os doadores e outro para a manutenção dos bancos de sangue. $\mathrm{O}$ diferencial da proposta foi o uso de técnicas de gamification, pois possui um potencial para promover maior engajamento dos doadores.

O BloodSys [Severo e Santos 2018] explorou as alternativas para auxílio ao processo de coleta de sangue, implementando procedimentos para otimização do tempo de espera dos doadores e integrando as etapas visando a redução de falhas durante o processo de doação de sangue. Contudo, o aplicativo ainda era um protótipo.

Já Silva, Rodrigues e Oliveira (2017) desenvolveram um sistema colaborativo que aproximou doadores e não doadores dos hemocentros através de eventos e conteúdos para divulgação por meio de um aplicativo móvel com integração nas redes sociais. Mais uma vez, esse aplicativo ainda seria validado como um protótipo inicial. Outra solução encontrada foi o aplicativo Donar [Dias e Albuquerque 2018], que objetivou conectar doadores aos locais de doação, levando em consideração a distância. Porém, o projeto ainda buscava parcerias para entrar em operação, diferentemente da proposta do presente artigo, que alcançou resultados concretos de implantação.

\section{Metodologia}

Inicialmente, o desenvolvimento do produto inovador DOE + foi direcionado com foco em um projeto de extensão de natureza tecnológica, pois envolveu o atendimento às demandas técnicas do HEMOAL com o intuito de contribuir em ações de diagnóstico, análises e implantação de serviços tecnológicos na área de plataformas móveis.

Assim, o proceder metodológico do projeto envolveu basicamente três etapas: (1) desenvolvimento e teste do aplicativo DOE+ para avaliar a aderência ao contexto 
organizacional do Hemocentro; (2) realização de visitas técnicas e entrevistas junto aos gestores e técnicos do HEMOAL e da SESAU para levantamento do processo da doação de sangue; (3) realização de treinamento e capacitação dos técnicos do HEMOAL para operacionalização do aplicativo.

As tecnologias computacionais adotadas foram: linguagem de programação Java, plataforma Android utilizando o plug-in ADT (Android Development Tools), ambiente Eclipse e o banco de dados PostgreSql. Também foi adaptado para uma versão do sistema operacional $i O S$ da Apple $e^{\complement}$. Já a interface web foi implementada em ambiente Ubuntu 14.04, usando o framework Ruby on Rails e banco de dados PostgreSQL. Para as notificações push entre a versão web e a mobile foi adotado o Google CloudAPI. O ambiente de desenvolvimento utilizou controle de versão do Ruby Version Manager. Ademais, dois frameworks auxiliares também foram adotado: Materialize para o frontend da aplicação web e o Passenger, que é um plugin do Apache para código Ruby.

\section{Resultados}

Durante os 3 (três) anos de uso, o produto de inovação tecnológica DOE+ possibilitou aos usuários-doadores do HEMOAL acesso a informações em tempo real sobre o tipo sanguíneo com níveis baixos no banco de sangue, além de notificações sobre novas campanhas e agendamento de horários específicos para doação no hemocentro. As principais funcionalidades implementadas para os usuários do $\mathrm{DOE}+$ foram as seguintes: visualização e notificação de campanhas institucionais; cadastro de novos doadores; agendamentos de doações em horários pré-definidos; visualização do nível dos estoques de sangue; notificação de doadores quando o estoque do seu tipo de sangue estiver baixo; compartilhamento via redes sociais $\left(\right.$ Facebook $^{\odot}$ e Twitter $^{\mathscr{O}}$ ); disponibilização de um dashboard com gráficos ilustrando a quantidade de doações.

Figura 1 - Telas de abertura, campanhas e agendamento do aplicativo
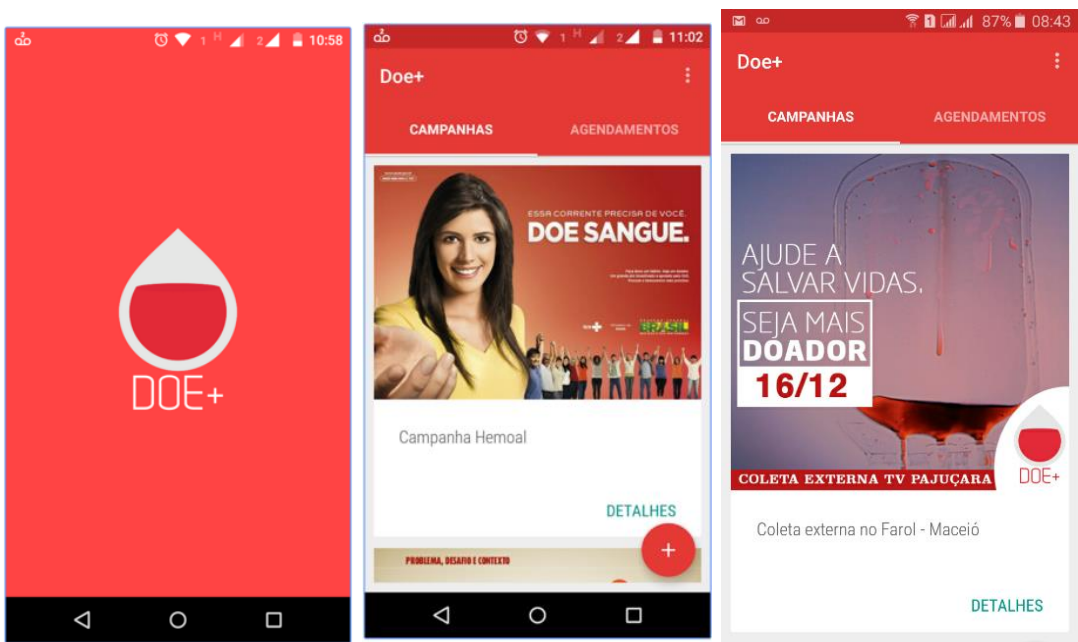

Assim, o aplicativo possibilitava ao usuário visualizar as campanhas institucionais e ser notificado quando as mesmas fossem cadastradas pelo HEMOAL (Figura 1). Estas funcionalidades foram alcançadas após a implantação e a oficialização do app pela diretoria do hemocentro, com o intuito de privilegiar os doadores que fizessem seus agendamentos de doação via smartphone. A Figura 2 ilustra as telas do 
app com os detalhes do agendamento do usuário (dia, horário e local) e com a opção de compartilhamento de uma campanha via redes sociais.

Figura 2 - Telas com detalhes do agendamento e compartilhamento em redes sociais do aplicativo
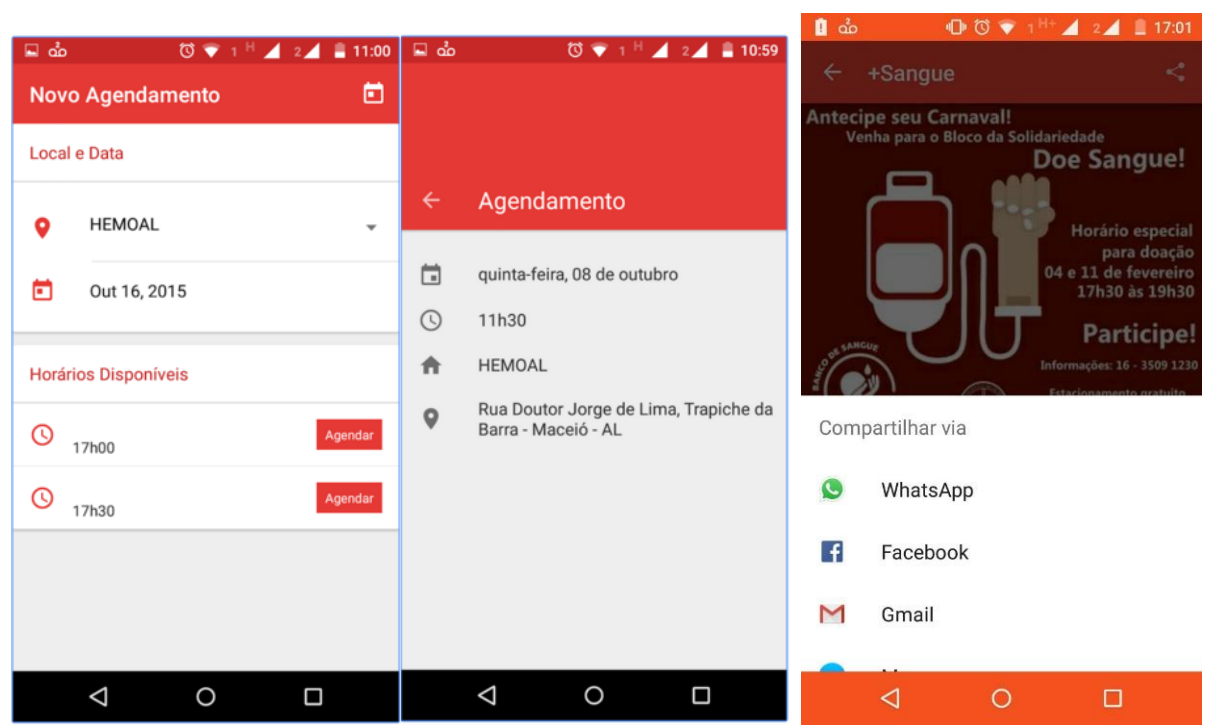

Também foi desenvolvido um software web com uma área de administração de acesso exclusivo dos técnicos do HEMOAL. Na tela inicial da versão web é possível visualizar um painel online (dashboard) que resume graficamente as informações sobre os quantitativos de agendamentos e doações (Figura 3), além de uma curva estatística com o número de downloads na loja virtual.

Figura 3 - Dashboard da interface administrativa com os números atingidos

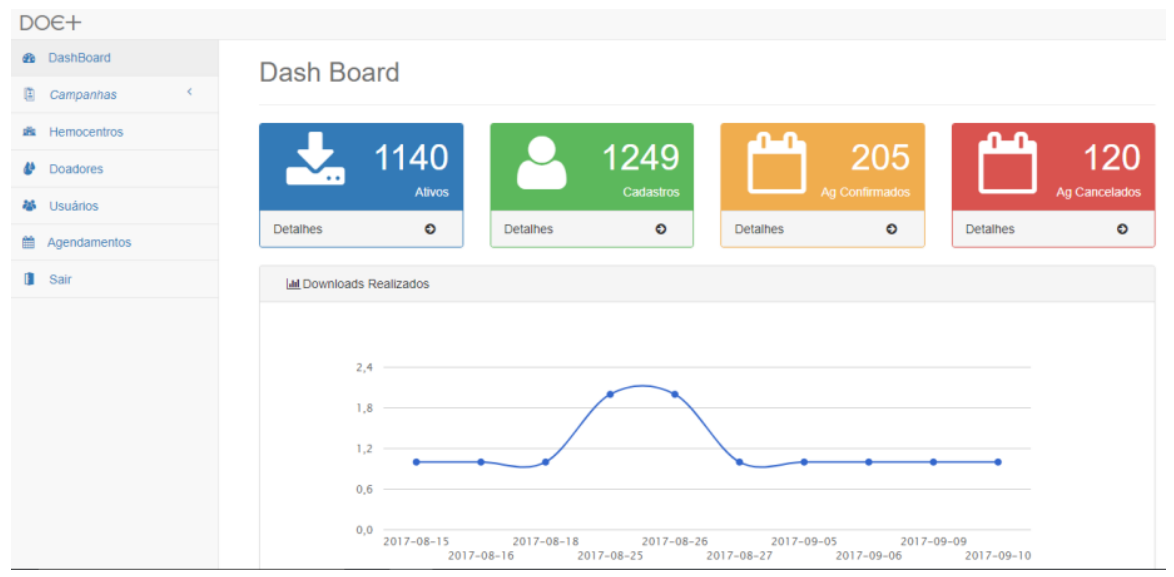

\section{Discussões}

Em um período anterior à implantação do $\mathrm{DOE}+$, foi possível perceber que as ações para captação de doadores alagoanos se desenrolava em ambientes virtuais inapropriados e não sistematizados para tal fim, como postagens em grupos no Facebook $^{\odot}$ e através de mensagens no WhattsApp ${ }^{\odot}$, com pouco direcionamento. 
Nos primeiros dois meses de uso o aplicativo alcançou 1.050 downloads, 576 cadastros de usuários e 106 agendamentos de doações. Após três anos de uso, o DOE+ atingiu mais de 3.000 downloads, 1.432 usuários ativos e 420 agendamentos de doação confirmados. Ressalta-se no gráfico ilustrado na Figura 3 anterior que o aumento de downloads do aplicativo na loja virtual no mês de agosto de 2017 foi fruto de uma campanha publicitária veiculada nas mídias locais pelo HEMOAL. Geralmente, em datas simbólicas e especiais, o aplicativo ganhava maior ênfase por parte do público e gerava tais picos de uso. As campanhas em 2017 e 2018 na Semana Nacional do Doador, por exemplo, foram outros eventos importantes que ajudavam no surgimento desses picos de download do app [Souza Júnior, Silva, Silva Júnior, 2019].

Analisando-se os dados gerados pelo uso do $\mathrm{DOE}+$, constatou-se um incremento de $47 \%$ na média semanal de atendimentos do HEMOAL após a implantação do aplicativo entre os anos de 2015 e 2017, incentivando inclusive a primeira doação de vários usuários jovens que aderiram à ferramenta digital. Esta percepção foi comprovada no momento da doação através de entrevistas empreendidas com os próprios doadores. A Figura 4 ilustra duas notícias na mídia sobre o DOE+, com destaque para a primeira usuária que agendou uma doação via aplicativo. Ela relatou que nunca havia doado sangue antes e foi estimulada pela campanha de lançamento do aplicativo para dispositivos móveis. Já a segunda notícia ilustrada na Figura 4 enfatiza o número de downloads nos primeiros dias do lançamento, que alcançou 750 instalações em dispositivos móveis através da loja virtual do Google ${ }^{\mathbb{C}}$.

\section{Figura 4 - Repercussão espontânea na mídia sobre o aplicativo DOE+}

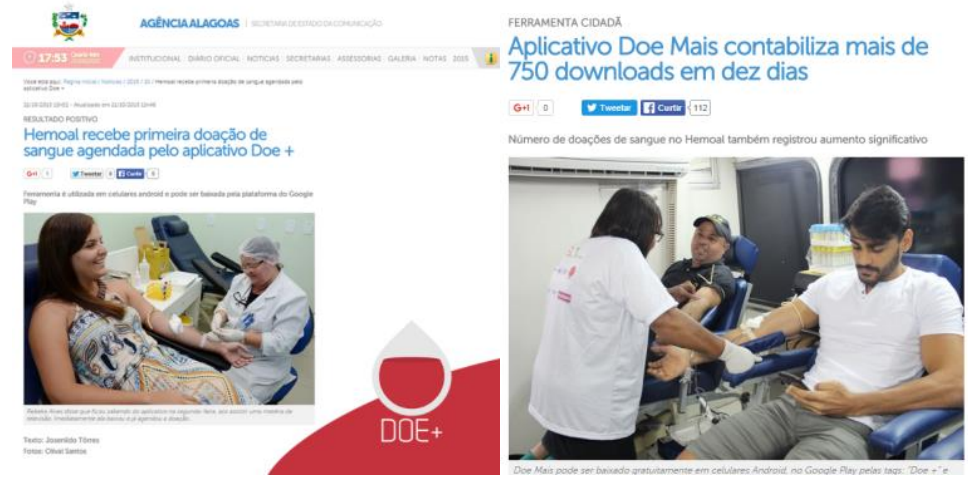

O perfil mais frequente dos usuários cadastrado no DOE+ foi do gênero feminino, com idade entre 18 e 23 anos e ensino médio completo. Este dado contrasta com estudos da Agência Nacional de Vigilância Sanitária (ANVISA), que verificou os homens como os usuários que mais procuram os serviços de hemoterapia para realizar uma doação de sangue do que as mulheres. Em relação ao tipo de doação, o maior número realiza a doação espontânea.

Os resultados alcançados demonstraram que o uso de tecnologias digitais voltado para este tipo de causa pode ser um instrumento essencial para que mudanças importantes aconteçam na sociedade. Conforme frisou Silva (2016), os estudos sobre ressignificação da doação de sangue é um processo lento, entretanto, quando pessoas que doam e usam apps compartilham com seus amigos, os quais podem vir a doar também, está se formando uma rede solidária capaz de salvar muito mais vidas. 
Araújo, Feliciano e Mendes (2011) ressaltaram que a grande maioria dos doadores de repetição (aqueles com intervalo de tempo entre as duas últimas doações igual ou inferior a 13 meses) ainda não está próximo do número máximo de doação anual. Portanto, torna-se fundamental focar a atenção neste grupo mais receptivo para aumentar o número de doações. É possível gerar relatórios estratégicos no DOE+ para identificar tais grupos potenciais e então planejar campanhas um pouco mais efetivas.

\section{Conclusões}

Em Alagoas, a deficiência na captação e fidelização de doadores de sangue também é um problema crônico, tal como nos centros de coleta de sangue em todo país, sendo que ao mesmo tempo é imprescindível conscientizar os doadores para a manutenção dos níveis de estoque de bolsas sanguíneas nos hemocentros.

O objetivo do aplicativo DOE+ foi contribuir com a sociedade alagoana a partir da criação e viabilização de um projeto que impactasse diretamente na vida do cidadão, além de ter sido uma ideia desenvolvida na academia que foi transferida para o setor público.

A solução tecnológica desenvolvida possibilitou que o HEMOAL se tornasse o primeiro hemocentro público com agendamento de doação de sangue via dispositivos móveis, pois se verificou que a cultura predominante nos hemocentros brasileiros ainda era o uso de agendamento por telefone ou via formulários online nos próprios websites.

No que tange a mudança de vida dos cidadãos, foi possível com o apoio da direção do HEMOAL, que uma das 6 (seis) cadeiras de doação existentes no hemocentro estivesse reservada no período que a solução esteve ativa para 0 atendimento exclusivo dos usuários que realizavam o agendamento usando o aplicativo DOE + , tornando-se um marco para aquele hemocentro.

Por fim, ocorreu a transferência da tecnologia desenvolvida para a SECTI, sendo finalizada em 2018, através da assinatura de um Acordo de Cooperação TécnicoCientífico entre os órgãos parceiros. Também foi obtido o registro de software junto ao Instituto Nacional da Propriedade Industrial (INPI), como Programa de Computador, sob número BR512016001276-5. Como reconhecimento do mérito social da solução, o projeto foi premiado na Mostra Tecnológica do Congresso Norte-Nordeste dos Institutos Federais (CONNEPI), que teve como tema "Inovação e Empreendedorismo".

\section{Referências}

Araújo, F. M. R., Feliciano, K. V. O. e Mendes, M. F. M. (2011) “Aceitabilidade de doadores de sangue no hemocentro público do Recife, Brasil". Ciência e Saúde Coletiva (online), Rio de Janeiro, v.16, n.12, p. 4823-32.

Baumgarten, M. (2006). “Tecnologias sociais e inovação social”. In A. D. Cattani, e L. Holzmann (Org.), Dicionário de trabalho e tecnologia. Porto Alegre: Editora UFRGS.

BRASIL. Ministério da Saúde (2001) "Política Nacional de Sangue e Hemoderivados". Decreto $n^{\circ} 3.990$, de 30/10/2001. Brasília: Ministério da Saúde. 
Dias, G. L. e Albuquerque, M. L. (2018) "Desenvolvimento do Aplicativo Donar para Ampliar o Volume de Doações de Sangue No Brasil". Monografia (Bacharelado em Engenharia da Computação) - Centro Universitário de Anápolis, UniEVANGÉLICA.

ESTRATEGIA SAÚDE DIGITAL (2017) “Estratégia de saúde digital no Brasil digiSUS". Disponível em http://portalarquivos.saude.gov.br/images/pdf/2017/julho/12/Estrategia-e-saude-parao-Brasil.pdf.

Hora, M. T. S. (2008) "Doação de sangue: o que revelam os dados do Hemocentro de Alagoas". Monografia (Especialização em Gestão do Trabalho em Saúde) Universidade Federal de Alagoas - UFAL.

Moura, A. (2009) "Geração Móvel: um ambiente de aprendizagem suportado por tecnologias móveis para a Geração Polegar”. In P. Dias, A. J. Osório (Org.), Anais da VI Conferência Internacional de TIC na Educação Desafios. Braga, 2009.

Oliveira, T. R. e Rodrigues Costa, F. M. (2012) "Desenvolvimento de aplicativo móvel de referência sobre vacinação no Brasil". Journal of Health Informatics, v.4, ed. 1.

Sojka, E. N. (2008) "The blood donation experience: self-reported motives and obstacles for donations blood". Vox Sanguinis, v.94, n. 1.

Saccol, A. Z. e Reinhard, N. (2007) "Tecnologias de informação móveis, sem fio e ubíquas: definições, estado-da-arte e oportunidades de pesquisa". Revista de Administração Contemporânea, v.11, n.4.

Ouhbi, S., Fernandez-Aleman, J.L., Toval, A., Idri, A. e Pozo, J.R. (2015) "Free Blood Donation Mobile Applications". Journal of Medical Systems, v39, n.52.

Orlando Silva Júnior, T. A. R. e Pereira, A. C. M. (2017) "PartiuDoarSangue - Uma Plataforma Web e Aplicativo Mobile para Captação e Gestão Inteligente de Doação de Sangue e Hemocomponentes". In: XXIII Simpósio Brasileiro de Sistemas Multimídia e Web - WEBMEDIA: Workshops e Pôsteres. Anais. Gramado-RS.

Severo, C. E. P. e Santos, H. M. (2018) "BloodSYS: controlando o processo de doação de sangue para hemocentros". Revista Eletrônica de Sistemas de Informação e Gestão Tecnológica, v.9, n.2.

Silva, A. L. (2016) "Boação: um estudo para o desenvolvimento de um aplicativo móvel facilitador para doação de sangue". Monografia (Graduação em Design) Universidade do Vale do Taquari - Univates.

Silva, R. e Barbosa de Araujo, D. (2017) "Proposta de um sistema de apoio à doação sanguínea baseado em gamificação". Revista de Engenharia e Pesquisa Aplicada, v.2.

Silva, A. O., Rodrigues, L. L. e Oliveira, R. R. (2017) "Sistema Colaborativo para Captação de Doadores de Sangue". In: Simpósio Brasileiro de Sistemas Colaborativos - SBSC. Anais. São Paulo-SP.

Souza Júnior, M. F.; Silva, N. R. B.; Silva Júnior, E. R. (2019) "DOE+: solução multiplataforma para incentivar a doação na hemorrede de Alagoas". In: Maria Rosa da Silva; Claudio Fernando Rodrigues Soriano; Claudio José dos Santos Júnior. (Org.). Hemoterapia Essencial. 1ed. Arapiraca: EDuneal. 